\title{
Side-by-side analysis of alternative approaches on multi-level RNA-seq data
}

\author{
IRINA MOHORIANU
}

\begin{abstract}
${ }^{1}$ School of Biological Sciences, University of East Anglia, Norwich Research Park, Norwich, NR4 7TJ, United Kingdom.

${ }^{2}$ School of Computing Sciences, University of East Anglia, Norwich Research Park, Norwich, NR4 7TJ, United Kingdom.
\end{abstract}

\#Corresponding author: i.mohorianu@gmail.com

Running title: Alternative approaches for multi-level RNA-seq data

The author has withdrawn this manuscript due to overlap with a different publication. Therefore, the author does not wish this work to be cited as reference for the project. If you have any questions, please contact the corresponding author. 